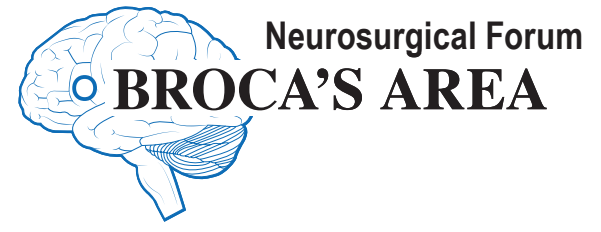

\section{Critical review of a scientific manuscript: a practical guide for reviewers}

\author{
Asdrubal Falavigna, MD, PhD, ${ }^{1}$ Michael Blauth, $\mathrm{MD},{ }^{2}$ and \\ Stephen L. Kates, MD³ \\ ${ }^{1}$ Neurosurgical Department, Caxias do Sul University, Caxias do Sul, \\ Brazil \\ 2Department of Trauma Surgery, Medical University Innsbruck, Austria \\ ${ }^{3}$ Department of Orthopaedic Surgery, Virginia Commonwealth University, \\ Richmond, Virginia
}

$\mathrm{P}$ EER reviewers have significant responsibilities toward authors, editors, and readers, providing some measure of "quality control" for published research using a fair and transparent critical assessment of the research. ${ }^{13}$ The reviewer can detect bias, unsatisfactory study design, and ethical problems that may threaten the research, and provide feedback to the authors. The critical assessment of the evidence and validity of the scientific publication enables the editor to accept, reject, or revise the manuscript, minimizing the authors' complaints if the paper is rejected. Even in those cases, the appropriate revision gives the author the chance to reorganize the article to resubmit it to another journal. ${ }^{6}$

Challenges of the peer-review process are: 1) the increasing need for reviewers due to an increasing number of peer-review requests, because promotions are obtained based on the number of publications or "publish or perish" syndrome and due to various online and hard copy publishers,${ }^{14}$ 2) increasing demands from the reviewers' primary jobs, related to clinical demands, financial constraints, or more time spent on their research program; and 3) heterogeneous quality of reviews and different methods used to analyze the manuscript, sometimes because of insufficient training. ${ }^{1,8}$ Most journal reviewers acquire the skills and knowledge to perform a manuscript review through their clinical expertise and their own ex- perience in critically appraising the literature. If an individual performs an inadequate review, it is likely that his or her service will not be requested again. Sometimes an inadequate review is not the reviewer's fault, but is due to insufficient formal training provided by the journals to establish standard methods to analyze the manuscript, or due to lack of information.

Even if the reviewers analyze the manuscript as though they themselves were submitting it, sometimes there is a lack of a comprehensive set of guidelines for all aspects of the review process, leading to an unsupported decision. ${ }^{10}$ To minimize this problem, the art of reviewing manuscripts should follow systematic scientific methods to enhance the quality and reduce the time spent on this practice. Systematic guidance minimizes the revision errors while the reviewers improve their practice. ${ }^{9}$

The aim of this paper is to develop a practical method for reviewing a manuscript, explaining how the topics need to be evaluated, and not just provide a checklist. We will focus on the peer review of research manuscripts submitted to scientific journals.

\section{Systematic Method for Manuscript Review}

A confusing or uninformative critique is not helpful to either the authors or the editor. If the reviewer disputes a point made by the authors, he or she should provide explicit justification for his or her argument. A critical justification for the strengths and weaknesses of the manuscript depends not only on the clinical expertise in a given subject area and the time available for the review but also on the use of standard guidelines during the revision process. Without a standard and systematic revision, there is a risk of missing important parts of the manuscript. The consequence can be a superficial review, with no real justification and support for the editor's decision.

In the introductory section of the manuscript, the reviewer will immediately develop an idea of the clinical question of the study to be validated. The introduction justifies the study and describes the objectives. It identifies current knowledge gaps and anticipates how the study results may close those gaps. The methodology used and 
the diagnostic hypothesis should also be described in this section. The hypothesis or superiority trial represents the objective of the study to reject the null hypothesis $(x=y)$ in favor of the alternative hypothesis ( $\mathrm{x}$ is different from $\mathrm{y}$ ).

\section{Validating the Clinical Question}

The reviewers can promote a general evaluation of the proposed research question by using the FINER criteria: Feasible, Interesting, Novel, Ethical, and Relevant. ${ }^{4}$ They must verify closely the research question or objective (aim) of the study because it is the most important part of the entire project. All the components of the study are strictly structured based on a clinical question: type of study, methodology applied, population studied, sample size calculation, time available, equipment, funding, instruments or questionnaire to measure the primary and secondary outcome or endpoint, and implementing the work. The questions that need to be answered by the reviewers are the following: 1) is there a clear, focused, and answerable study question; 2) is the study question innovative or relevant; 3) does the manuscript present an updated literature; 4) has the question already been answered in the literature; 5) does the study have the potential to advance scientific knowledge, influence clinical management and health policy, or provide some directions to future research; 6) does it matter; 7) what relevant information will the study add to the literature; and 8) is the paper clearly written and well organized?

\section{Methods}

The methods section of the article is the study design. A well-elaborated methods section may convince the reviewers of the validity of the study design, the reliability and competence of the research team, and thus the reproducibility of the results. If other researchers apply the same methods under the same conditions, the results should be similar.

This section is the fundamental part of the paper where the reviewer will analyze the internal and external validity of the study. To do that, the reviewer needs to understand the specific aspects of the methodology with greater refinement and precision using PICOT (Patient, Intervention, Comparative, Outcome, Timing, and Type of study), complemented with a verification of regulatory approval, examination of sample size, quality of patient allocation, maintenance of treatment, and presence of masking techniques. ${ }^{5}$ The systematic review suggested in the methods section is: patients $(\mathrm{P})$, regulatory approval, sample size calculation, allocation, intervention group (I), control group $(\mathrm{C})$, maintenance, outcome $(\mathrm{O})$, blinding techniques, time of study (T), and type of study (T).

\section{Patients or Population}

In this section, the reviewers should verify the eligibility criteria for inclusion and exclusion of the participants and establish the clinical or radiological characteristics of the patients who will become part of the study. The reviewer must identify if the group is homogeneous, if the characteristics of the patients are representative of those in the clinical question, and if it is acceptable to extrapolate the study data to the clinical question when the characteristics are not similar.

The authors have to describe how the confounding factors were controlled (exclusion criteria). For example, some of the variables controlled could be: demographic characteristics (age and sex), habits (smoker, drinker), use of medications (steroids), comorbidities (diabetes mellitus, morbid obesity, neoplasm), general conditions of quality of life (unemployed), pregnancy, previous surgeries, degree of severity of the disease, and early or late presentation. The adequate description of eligibility criteria allows the reproducibility of the study. The questions that need to be answered by the reviewers are the following: 1) are the eligibility criteria for inclusion and exclusion broad and clearly stated; 2) is the condition used in the selection clear, such as tests, scores, signs, and symptoms; 3) is the group homogeneous; 4) are the characteristics of the patients representative of those of the clinical question; 5) are the baseline characteristics reported; and 6) were patients similar at baseline in terms of demographics and comorbidity?

\section{Regulatory Approval: Reporting of Informed Consent and Ethics Committee Approval}

The reviewer should remember that there is no clinical study without disclosure of ethical protection, committee approval, and the patient's informed consent. Sometimes the journal requires that the clinical study be entered into a registry to be published. The best-known registry is probably www.clinicaltrials.gov, but there are other registries such as www.anzctr.org.au. By registering the study the authors undertake the commitment to avoid the situation in which if the results of a trial are negative, a decision has to be made to either not publish the results or delay publication for an unspecified time. ${ }^{3}$ Conflict of interest, funding information, and other support should be reported, if they exist. The reviewers need to answer the following: 1) if humans are studied, or human tissues or animals are involved, has ethics approval been obtained and is the study ethical; 2) is the paper in agreement with the standards of medical ethics; 3) is informed consent applied; 4) is the study registered; and 5) are there any conflicts of interest involving the authors?

\section{Sample Size Calculation}

Once the eligibility criteria for patients or a sample of the population has been described, it is necessary to examine whether the sample size of the study has been calculated. Sample size calculation is usually a requirement for ethics committee approval of a prospective study.

The studies that perform sample size calculation provide a clear indication of methodological quality, diligence, reasoning, and an increase of study power. The reasons for this are the need to recruit enough patients into the study to detect the anticipated effect, to minimize the chance of the study results being erroneous, and to use resources efficiently because every patient recruited into the study costs money. There is no guarantee that the statistically significant findings really represent true results in a prospective study without a sample size calculation. The conclusion 
can be: 1) there is no effect, or 2) the study was too small to detect a difference.

The reviewer needs to verify whether the manuscript describes the parameters used to calculate the sample size. The parameters are: 1) level of statistical significance, usually stated as $5 \%$; 2) the power of the study, usually set as 20\%; and 3) clinical parameters. The size of the clinical effect under investigation has an inverse relation with the sample size of the study: the larger the clinical effect, the greater are the chances of showing the difference in the investigation in a small sample size. Conversely, small clinical effects preclude the study of larger sample sizes.

A Type-I error ( $\alpha$ error) represents the "level of statistical significance of study." This happens when an apparently positive result may, in fact, be negative (false positive) and the null hypothesis is erroneously rejected. Although apparently effective in a clinical trial, a treatment may not be effective. A Type-I error is typically set as a study significance level of 5\% $(\alpha=0.05)$, the risk of 1 in 20 to see a false-positive result.

A Type-II error ( $\beta$ error) represents the "power of study." Although there is a real difference (= effect) between the groups, statistical testing may indicate no significant results. There is always a risk of missing a positive treatment effect (false negative). A Type-II error is typically set as a study power of $10 \%-20 \%(1-\beta=1-0.20=0.80$ [80\%]). For example, if you have $80 \%$ power in your sample size calculation, it means that if there is an effect on your study, the probability of not detecting it is as low as $20 \%$.

From a practical standpoint, the reviewer can follow the 50/50 rule (proportions): 50 events are needed in the control group for an $80 \%$ chance of finding a $50 \%$ reduction. ${ }^{7}$ For example, in a particular surgical spine technique there is a $12 \%$ rate of infection $(12 / 100$ or $24 / 200$ or $48 / 400$ or $50 / 417)$. The authors want to show an infection reduction of $50 \%$ with local use of topical vancomycin. The total sample size calculation for the control $(n=417)$ and treatment $(n=417)$ groups will be 834 patients. The questions that need to be answered by the reviewers are: 1) did the authors perform a sample size calculation; 2) do the authors mention the power of their study; 3 ) what level of significance was calculated (in \%); and 4) are the clinical parameters used to calculate clinical significance?

\section{Allocation}

Once the eligibility criteria for patients or population have been described and the sample size calculation performed, it is necessary to look at the type of distribution used, if applied.

Randomization. Randomization allows assigning patients to a particular treatment by chance, balancing known and unknown confounders equally to the study arms. In these cases, the investigators can interpret the differences in outcomes caused by the intervention and not by chance. For example, patients with comorbidities are likely to have even worse outcomes than patients without. If all patients with diabetes and other patients with complications were moved to the conservative arm, it would unbalance the study and make the results from the operative arm appear even better than they are.

Effective randomization depends on adequate eligibil- ity criteria for patients. It should be underscored that after designating a patient either to the experimental group or to the control group, they must remain in it until the end of the study, except when there are reasons justifying their removal.

It is important not only to say that the study was randomized but also to describe the randomization used to verify if it is adequate. The off-site randomization center reduces any influence from the researchers. Any system that can be guessed (such as using patient dates of birth) or influenced by an investigator who selects which envelope to pick to choose patients for their study, is not real randomization. When the envelope is the method used, it is recommended to provide a tamper-proof envelope to prevent having the treatment or diagnosis group chosen on purpose.

Randomization allows one to: 1) provide for comparable study groups; 2) balance known and unknown confounders equally to each study arm; 3 ) remove investigator bias and maintain internal validity; and 4) interpret the differences in outcomes caused by the intervention and not by chance. The questions that need to be answered by the reviewers are: 1) are patients allocated randomly to trial groups; 2) is the randomization described and detailed enough to verify its adequacy; and 3) can you think of any way in which someone could get around the randomization process described in the article?

\section{Intervention and Comparison}

Intervention is the drug, new surgical technique, diagnostic test, or risk (prognostic) factor for the outcome of interest that will be compared with conventional treatment. This must include specific technical aspects, such as necessary instruments, biological materials, or equipment; type of procedure, levels to be treated, need for training that will justify different performances, type of test, the protocol to apply it, and diagnostic criteria.

The comparison group is the current reference drug, test, or technique that is the gold standard for this type of disease or situation or patients without the prognostic factor of interest. This item will be the control group. Technical aspects and criteria for diagnosis or treatment of the disease must be outlined. When the research question involves safety, there is no comparison group. Questions to be answered by the reviewers are: 1) do the authors adequately describe the treatment (including postoperative protocol) for each treatment arm; 2) are the intervention and comparison sufficiently detailed to ensure their applicability; and 3) is the comparison useful for the clinical question?

\section{Maintenance}

The reviewer must pay attention not only to the eligibility criteria and the treatment or diagnosis performed in the intervention and control groups, but also the maintenance of the patients in those groups. It is important that the reviewer checks for equal treatment and makes sure that the patients are treated as equally as possible. For example, if one group of patients receives a course of physiotherapy while the other does not, you would expect the group in physiotherapy to show a better early outcome. Without 
having a comparable group to measure them against, the manuscript cannot conclude that the better outcomes observed were related to the surgery or the physiotherapy sessions.

The conclusion of the study can only be confirmed if the patients who are lost to follow-up during the study are accounted for. Many may have dropped out due to treatment-related complications. A dropout rate below $5 \%$ is excellent; if the rate is $5 \%-20 \%$, the results are questionable; a rate equal to or higher than $20 \%$ is considered unacceptable and poses serious threats to validity. It is important that the reviewer analyzes if the losses are equally distributed and have similar characteristics. Similarly, the crossover needs to be assessed.

Maintenance means to certify that the patients were: 1) treated equally and received identical care apart from the actual intervention; 2) compliant with the study and completed all the evaluations; and 3) compliant with the allocation group or crossover during the treatment. The reviewer can clearly understand the trial design, conduct, analysis, and interpretation, and assess the validity of its results if the manuscript includes the Consolidated Standards of Reporting Trials (CONSORT) statement flow diagram (Fig. 1). ${ }^{12}$ Questions to be answered by the reviewers are: 1) are patients treated equally and comparable apart from the intervention group; 2) how many patients dropped out during the study; 3) what is the dropout rate; 4) are the dropouts equally distributed and do they have similar characteristics; 5) how many patients completed all the evaluations; 6) how many patients crossed over during the study; and 7) is there a flow diagram reporting the quantity of patients eligible and included in the study, the number of subjects in each treatment arm, and how the study ended, by having the number and the reasons why patients left the study at various points in time?

\section{Outcomes}

The reviewer must clearly detect the primary and secondary outcomes or endpoints and how they were measured. The outcomes should be clinically relevant to quantify benefit, damage, or risk. While there might be statistical relevance in a finding, it may not be clinically significant for the patient's treatment.

The instruments or questionnaires used for measurement need to be objective, well described in the literature, and validated for the native language of the patient. The outcome of interest can be positive (percentage of fusion or satisfaction) or negative (complication or dissatisfaction). In diagnostic studies, the outcomes of interest are usually calculations of sensitivity, specificity, and positive and negative predictive value.

The data collection in a prospective method has greater credibility when the result and evolution over time are recorded. The manuscript should describe how many follow-up visits were performed and the time points used to measure the outcomes, e.g., pretreatment, posttreatment 1 month, 6 months, 1 year, 2 years, and 5 years.

Questions to be answered by the reviewers are: 1) are the outcomes clinically relevant or important to the patients; 2) are the instruments or questionnaires for measurement objective; 3) are the instruments or questionnaires for measurement well described in the literature to enable a correlation of the results; 4) are patient-reported outcomes used or just physiological process or radiographic measures; 5) are the evaluation instruments appropriate for the study and validated for the language of the country where they will be applied; 6) is the outcome assessor (evaluator) blinded; 7) how is the data collection performed (prospectively or retrospectively); and 8) how many follow-up visits are performed and how many time points are used to measure the outcomes?

\section{Blinding or Masking Techniques}

Blinding or masking techniques seek to keep the information hidden from the patient and/or the physician. This prevents study patients and investigators from determining the group to which the individual has been assigned after allocation. Blinding cannot always be implemented but should always be considered whenever a study is being planned. The reviewer needs to validate whether the masking methods used were performed adequately. Hence, they have to verify not only if blinding techniques are used but also who was blinded and how it was conducted.

Patients may do better or worse, depending on the group to which they belong, based on their own desires or prior knowledge (placebo effect). If it is impossible to mask the patient or the principal physician, generally at least the evaluator is blinded during the outcome assessment to avoid interviewer bias. It is not prudent for the physician who is performing the investigation to be the same person who conducts the outcome assessment (evaluator). The physician who is treating the patient may have a special interest in going on with the research and unintentionally modify the information or influence the experimental group differently from the control group. This is even more important when the outcome is not objective. Questions to be answered by the reviewers are: 1) is blinding used; 2) if blinding is used, who is blinded; 3) has the blinding process been described; 4) would it have been possible for the blinded person to realize which intervention was performed; and 5) if no blinding is reported, can you think of a way in which blinding could have been used in the study (e.g., blinding the outcome assessor, etc.)?

\section{Timing}

The minimum follow-up time must have been established to observe (or not observe) the expected outcome. In a surgical specialty, it is very important to have medium and long-term follow-up. The outcome of a surgical technique or treatment is often modified after the first year or even longer. The question to be answered by the reviewers is: was the timing for observation adequate to detect differences between the groups?

\section{Type of Study}

The reviewer needs to correlate the type of study selected, whether it be observational or interventional, with the possibility that it will answer the research question. The type of study indicates the level or quality of evidence and the accuracy of the information (Fig. 2). In a primary study the analysis is performed on only one measure, and in a secondary study the analysis is performed on several measures. 


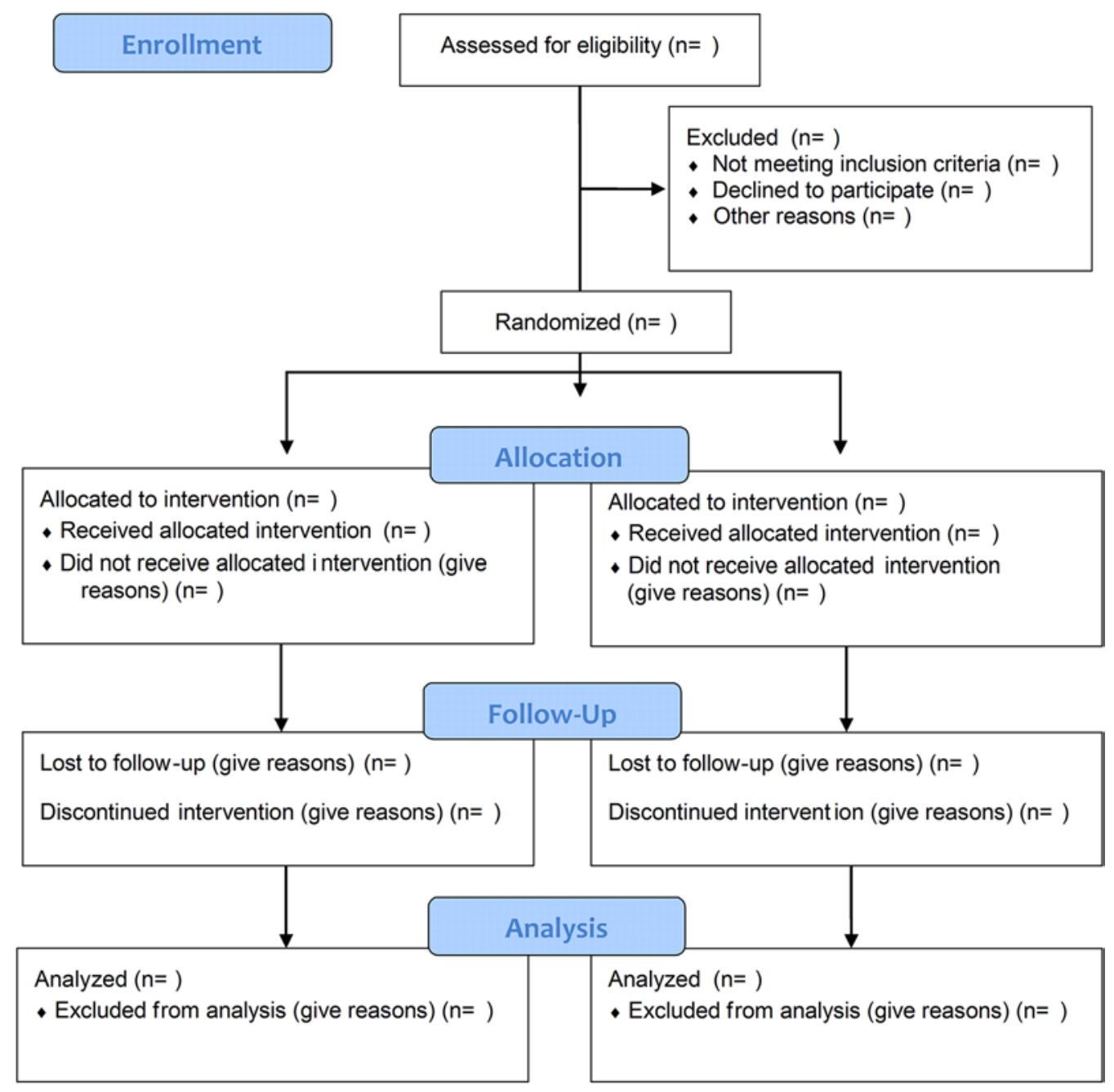

FIG. 1. CONSORT statement flow diagram. Figure is available in color online only.

Primary Studies. Primary studies can observe the patient evolution after therapy (observational study) or if the investigator performs an intervention (interventional study). The case series is a descriptive and noncomparative study, while the cross-sectional longitudinal study and randomized clinical trials are analytical and comparative studies.

Observational Studies. Observational studies can be classified as case series, cross-sectional, and longitudinal prospective cohort or longitudinal retrospective case-control studies. A case series is a descriptive study located at the base of the evidence pyramid. A cross-sectional study evaluates the risk factors and effect at a single point in time without any follow-up. It provides more evidence than case series and is located on the level of evidence (LOE) between the case series study and the longitudinal retrospective cohort study. A longitudinal study evaluates the patient at different times. The classification can be retrospective (case-control study) and prospective (cohort study). They are located on the level or quality of evidence between the cross-sectional studies and randomized clinical trial. In a retrospective cohort study, the outcome is measured at the end of the work, and the variables are verified in the database or patient records. In a prospective cohort study, the outcome and the measures are defined at the beginning of treatment and the patients are followed to verify if the disease is positive or not.

Interventional Studies. In an interventional study, the investigator promotes an intervention. Usually the randomization is used to locate the patient (randomized clinical trial) with a high quality of evidence. When the study has an intervention without randomization, it is defined as a nonrandomized controlled trial.

Secondary Studies. Secondary studies are located at the top of the quality of evidence and are defined as systematic reviews and meta-analyses. The review of systematic or meta-analysis manuscripts needs to follow the acronym FAITH, which represents: 1) find appropriate studies; 2) appraise selected studies; 3 ) include only valid studies; 4) total-up (synthesize) appropriately; and 5) heterogeneity adequately addressed.

Levels of Evidence of Studies. Twenty years ago, Suzanne Fletcher and Dave Sackett generated LOEs for rank- 


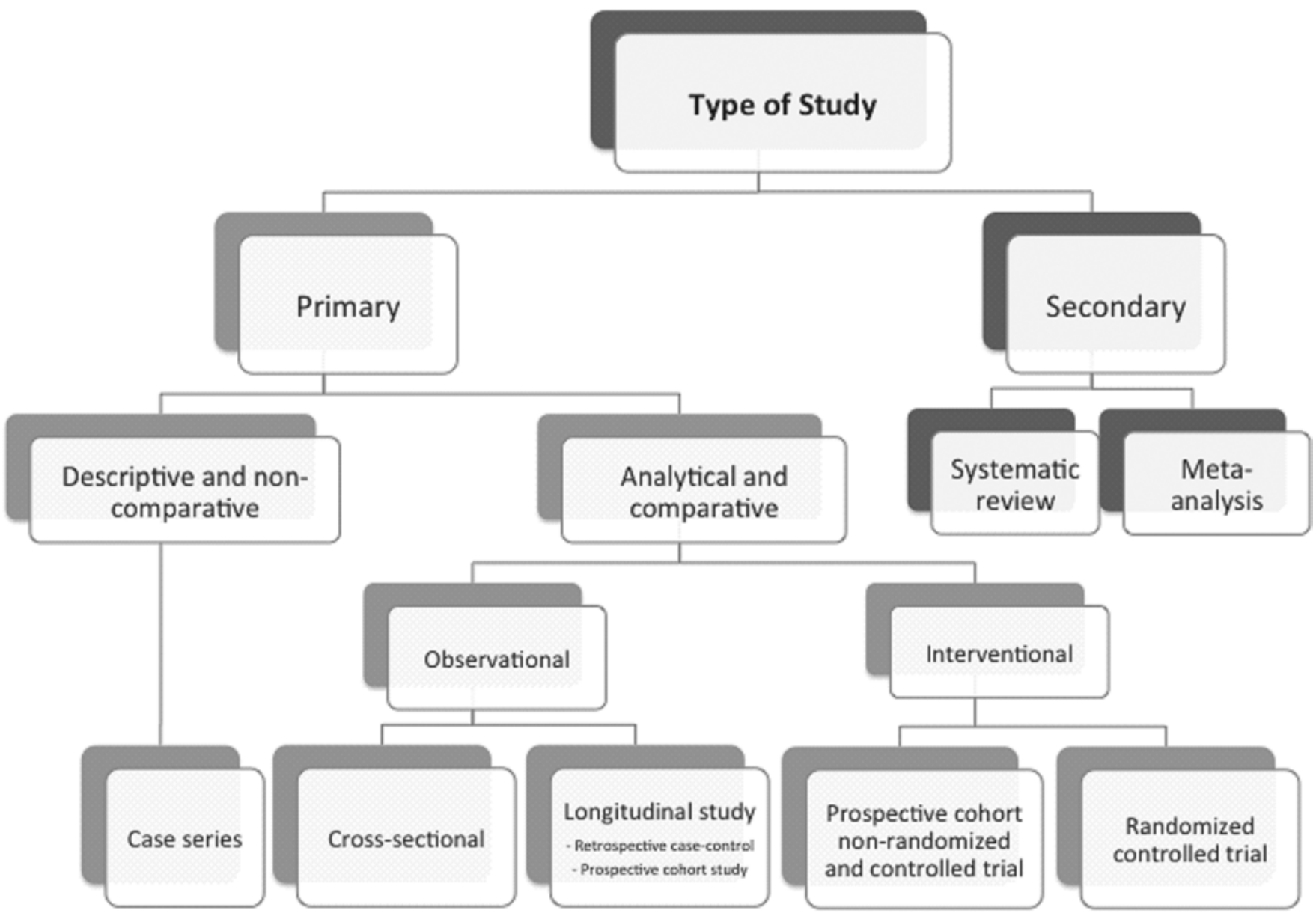

FIG. 2. Types of primary and secondary studies.

ing the validity of evidence about the value of preventive maneuvers and then attached them as "grades of recommendation" to the advice in the report for the Canadian Task Force on the Periodic Health Examination. ${ }^{2}$ The Centre for Evidence-Based Medicine (CEBM) developed Levels for Therapy, Prevention, Aetiology, and Harm (http:// www.cebm.net/ocebm-levels-of-evidence/) (Table 1).

The grades of recommendation were defined as A, B, C, and D. Grade A is consistent with Level 1 studies. Grade B is consistent with Level 2 or 3 studies or extrapolations from a Level 1 study. Grade $\mathrm{C}$ is when there are Level 4 studies or extrapolations from Level 2 or 3 studies. Grade D is when there is Level 5 evidence or troublingly inconsistent or inconclusive studies of any level. "Extrapolations" are where data are used in a situation that has potentially clinically important differences from the original study situation. Questions to be answered by the reviewers are: 1) what was the type of study, and 2) was the study design appropriate for the research question?

\section{Statistical Analysis and Data Presentation}

The intention-to-treat analysis analyzes the patient according to which treatment they were assigned, even though they have received another treatment than the originally randomized one (crossover). Other options will be according to which treatment they received, named astreatment analysis.

Reproducibility is an important element of clinical research. It is necessary that others be able to reproduce experiments to test the results. Clinical researchers should describe the interventions used, the statistical tests used, as well as the results obtained so that other researchers can examine their results for methodological rigor and allow reproducibility. Questions to be answered by the reviewers are: 1) did the authors describe the statistical software used; 2) did the authors explain what statistical models were used; 3) did the authors provide sufficient numerical information for someone to check the statistics that were calculated; 4) are patients analyzed as randomized (intent to treat) or according to the treatment (as treated); 5) could presentation of the results be improved and do they answer the question; 6) how large is the treatment effect; 7) how precise is the estimate of the treatment effect; 8) is the baseline information table described; 9) do the figures and tables have important information and describe the data accurately; and 10) are they consistent, e.g., are bars in charts the same width, are the scales on the axes logical?

\section{Discussion}

The main objective of the discussion is to place the re- 
TABLE 1. Levels of evidence according to the Centre for Evidence-Based Medicine for Therapy, Prevention, Aetiology, and Harm

\begin{tabular}{cl}
\hline LOE & Study Type \\
\hline $1 a$ & Systematic reviews (with homogeneity) of randomized controlled trials \\
\hline $1 \mathrm{~b}$ & Individual randomized controlled trials (with narrow confidence interval) \\
\hline $1 \mathrm{c}$ & All or none randomized controlled trials \\
\hline $2 \mathrm{a}$ & Systematic reviews (with homogeneity) of cohort studies \\
\hline $2 \mathrm{~b}$ & Individual cohort study or low-quality randomized controlled trials (e.g., <80\% follow-up) \\
\hline $2 \mathrm{c}$ & "Outcomes" research; ecological studies \\
\hline $3 a$ & Systematic review (with homogeneity) of case-control studies \\
\hline $3 \mathrm{~b}$ & Individual case-control study \\
\hline 4 & Case series (and poor quality cohort and case-control studies) \\
\hline 5 & Expert opinion without explicit critical appraisal, or based on physiology, bench research, or "first principles" \\
\hline
\end{tabular}

search findings in the context investigated, explain their meaning, and emphasize their importance as compared with the findings and the opinion of authors who have already researched the field or topic. The discussions should be constructed hierarchically: start with the most important (primary) result and contrast it with the available evidence. Then address the secondary objectives and their results in paragraphs that follow. Whenever possible, the content should be put into the same divisions or subheadings used in the methods and results sections.

\section{Internal and External Validity}

Once the critical review of each of the parts of a scientific work is finished, the most complicated part is to identify if the results are valid and credible and if the study can be clinically or surgically applied to everyday work.

\section{Internal Validity or Credibility of Study}

The internal validity or credibility of the study is the capacity of the results to support a cause-effect relationship. In other words, the observed difference in outcome between groups is attributable only to the effect of the intervention under investigation. The internal validity is assessed by adequacy of allocation sequence, concealment of allocation sequence, blinding, the balance of prognostic factors, intention-to-treat analysis, and completeness of follow-up.

The reviewers need to have the ability to recognize the relationship between an explanatory factor and an outcome and to identify other variables (confounding factors) or systematic error (bias) that can distort the results. Confounding factors and study bias are different problems in the study that lead to an inaccurate estimate (underestimate or overestimate) of the true association between exposure and an outcome.

Confounding Factors. A confounding factor occurs when the effect or association between an exposure and outcome is distorted by the presence of another variable (confounding variable), resulting in a "mixing of effects." 11 Recommendations to the authors include: control several confounding factors during design and implementation of the study, i.e., by performing the sample size calculation, adequate allocation, having a control group, and using masking procedures.

Study Bias. Bias is a systematic error in the design, re- cruitment, data collection, or measurement that results in a mistaken estimation of the true effect of the exposure and the outcome. The reviewer must be aware of potential study bias because the smaller the bias the higher the chance of finding the truth. The types of study biases are selection, allocation or indication, treatment, and measurement or detection bias.

In selection bias, the bias can occur when enrolled patients are not representative or there is a misdiagnosis of the target patient population or retention of participants. Recommendations to the authors include: describe all the eligibility criteria, include all patients, and maintain tracking and communications with them.

Allocation or indication bias occurs when patient allocation to the treatment is influenced by prognostic factors or physician's willingness. Recommendations to the authors include: some type of allocation needed; the best is patient allocation by a computer-generated procedure.

Treatment bias occurs when the patient maintenance and compliance with the study are not appropriate, resulting in different postoperative patient care between the groups and losses of follow-up or crossover between the therapy arms. Recommendations to the authors are: blinding the clinical staff involved in the postoperative treatment and standardizing the pre- and postoperative routine care, such as physiotherapy and medication drugs and dosage.

Measurement or detection bias occurs when patient follow-up and outcome assessment differ between the groups or an inaccurately calibrated instrument creates a systematic error. Recommendations to the authors include: blinding the evaluator, use of objective and not subjective instruments to measure the endpoints, and standardized follow-up visits, ensuring the same chances to detect complication. Questions to be answered by the reviewers are: 1) was the study performed according to the original protocol; 2) are the results valid and near the truth; 3) are there selection systematic errors or bias; 4) are there allocation systematic errors or bias; 5) are there treatment systematic errors or bias; 6) are there measurement systematic errors or bias; 7) are there many confounding factors; 8) is there an appropriate sample size calculation, adequate allocation, good study design, blinding techniques, intention-totreat analysis, and completeness of follow-up; 9) are the methods described clearly enough for other researchers to 
TABLE 2. The reviewer must be able to make a checklist and the following questions should be kept in mind and answered before sending the review to the editor

\begin{tabular}{|c|c|c|}
\hline Variable & Essential Elements to Determine the Credibility of the Article & $\begin{array}{l}\text { Comments } \\
\text { (yes or no) }\end{array}$ \\
\hline $\begin{array}{l}\text { Validating the clini- } \\
\text { cal question of } \\
\text { the study }\end{array}$ & $\begin{array}{l}\text { Is there a clear, focused, and answerable study question? } \\
\text { Is the study question innovative or relevant? } \\
\text { Does the manuscript present an updated literature? } \\
\text { Has the question already been answered in the literature? } \\
\text { Does the study have the potential to advance scientific knowledge, influence clinical management and health } \\
\quad \text { policy, or provide some directions to future research? } \\
\text { Does it matter? } \\
\text { What relevant information will the study add to the literature? } \\
\text { Is the paper clearly written and well organized? }\end{array}$ & \\
\hline PICOT: "Patient" & $\begin{array}{l}\text { Are the eligibility criteria for inclusion and exclusion broad and clearly stated? } \\
\text { Is the condition used in the selection clear: tests, scores, signs, and symptoms? } \\
\text { Is the group homogeneous? } \\
\text { Are the characteristics of the patients representative of those of the clinical question? } \\
\text { Are the baseline characteristics reported? } \\
\text { Were patients similar at baseline in terms of demographics and comorbidity? }\end{array}$ & \\
\hline $\begin{array}{l}\text { Sample size } \\
\text { calculation }\end{array}$ & $\begin{array}{l}\text { Did the authors perform a sample size calculation? } \\
\text { Do the authors mention the power of their study? } \\
\text { What level of significance do they calculate (in \%)? } \\
\text { Are the clinical parameters used to calculate clinical significance? }\end{array}$ & \\
\hline Allocation & $\begin{array}{l}\text { Are patients allocated randomly to trial groups? } \\
\text { Is the randomization described and detailed enough to verify its adequacy? } \\
\text { Can you think of any way in which someone could get around the randomization process described in the article? }\end{array}$ & \\
\hline $\begin{array}{l}\text { PICOT: "Inter- } \\
\text { vention" and } \\
\text { "Comparative" }\end{array}$ & $\begin{array}{l}\text { Do the authors adequately describe the treatment (including postoperative protocol) for each treatment arm? } \\
\text { Are the intervention and comparison sufficiently detailed to ensure their applicability? } \\
\text { Is the comparison useful for the clinical question? }\end{array}$ & \\
\hline PICOT: "Outcome" & $\begin{array}{l}\text { Are the outcomes clinically relevant or important to the patients? } \\
\text { Are the instruments or questionnaires for measurement objective? } \\
\text { Are the instruments or questionnaires for measurement well described in the literature to enable a correlation of } \\
\text { the results? } \\
\text { Are patient-reported outcomes used or just physiological or radiographic measures? } \\
\text { Are the evaluation instruments appropriate for the study and validated for the language of the country where they } \\
\text { will be applied? } \\
\text { Is the outcome assessor (evaluator) blinded? } \\
\text { How is the data collection performed (prospectively or retrospectively)? } \\
\text { How many follow-up visits are performed and how many time points are used to measure the outcomes? }\end{array}$ & \\
\hline $\begin{array}{l}\text { Masking or blind- } \\
\text { ing techniques }\end{array}$ & $\begin{array}{l}\text { Is blinding used? } \\
\text { If there is blinding, who is blinded? } \\
\text { Has the blinding process been described? } \\
\text { Would it have been possible for the blinded person to realize which intervention was performed? } \\
\text { If no blinding is reported, can you think of a way in which blinding could have been done in the study (e.g., blinding } \\
\text { the outcome assessor, etc.)? }\end{array}$ & \\
\hline
\end{tabular}


» CONTINUED FROM PAGE 319

TABLE 2. The reviewer must be able to make a checklist and the following questions should be kept in mind and answered before sending the review to the editor

\begin{tabular}{|c|c|c|}
\hline Variable & Essential Elements to Determine the Credibility of the Article & $\begin{array}{l}\text { Comments } \\
\text { (yes or no) }\end{array}$ \\
\hline $\begin{array}{l}\text { PICOT: "Time of } \\
\text { follow-up" }\end{array}$ & Was the timing for observation adequate to detect differences between the groups? & \\
\hline $\begin{array}{l}\text { PICOT: "Type of } \\
\text { study" }\end{array}$ & $\begin{array}{l}\text { What was the type of study? } \\
\text { Was the study design appropriate for the research question? }\end{array}$ & \\
\hline $\begin{array}{l}\text { Statistical analysis } \\
\text { and data pre- } \\
\text { sentation }\end{array}$ & $\begin{array}{l}\text { Did the authors describe the statistical software used? } \\
\text { Did the authors explain what statistical models were used? } \\
\text { Did the authors provide sufficient numerical information for someone to check the statistics that were calculated? } \\
\text { Are patients analyzed as randomized (intent to treat) or according to the treatment (as treated)? } \\
\text { Could presentation of the results be improved and do they answer the question? } \\
\text { How large is the treatment effect? } \\
\text { How precise is the estimate of the treatment effect? } \\
\text { Is the baseline information table described? } \\
\text { Do the figures and tables have important information and describe the data accurately? } \\
\text { Are they consistent, e.g., are bars in charts the same width, are the scales on the axes logical? }\end{array}$ & \\
\hline Internal validity & $\begin{array}{l}\text { Was the study performed according to the original protocol? } \\
\text { Are the results valid and near the truth? } \\
\text { Are there selection systematic errors or bias? } \\
\text { Are there allocation systematic errors or bias? } \\
\text { Are there treatment systematic errors or bias? } \\
\text { Are there measurement systematic errors or bias? } \\
\text { Are there many confounding factors? } \\
\text { Are there sample size calculation, adequate allocation, good study design, blinding techniques, intention-to-treat } \\
\text { analysis, and completeness of follow-up? } \\
\text { Are the methods described clearly enough for other researchers to replicate? } \\
\text { Are the study limitations described in the discussion? } \\
\text { Do the data justify the conclusions? }\end{array}$ & \\
\hline External validity & $\begin{array}{l}\text { Does the study evaluate efficacy or effectiveness of a technique or product? } \\
\text { Is there enough information to reproduce the study elsewhere? } \\
\text { Can I see the limitations and strengths if I decide to reproduce the study? } \\
\text { Are the results applicable, easy to implement, and can they probably modify the evolution of diseases? } \\
\text { Can I generalize this study to my everyday work and my patients? } \\
\text { Will the results help me in caring for my patient? }\end{array}$ & \\
\hline
\end{tabular}

replicate; 10) are the study limitations described in the discussion; and 11) do the data and results justify the authors' conclusions?

\section{External Validity or Clinical Applicability}

The reviewer should ask if the results will help doctors care for their patients in the clinical setting. It is important for the reviewer to understand the difference between efficacy and effectiveness of the results. Efficacy of a technique or product arises from the results obtained by a given center or professional who is outstanding on this subject. Usually, the randomized trial is the appropriate study to verify efficacy because it provides greater control of the possible confounding variables. Effectiveness is the capacity to reproduce and obtain the same results within the medical community, using different centers and with professionals who have distinct degrees of experience. The observational study is generally used, because it selects patients with more heterogeneous characteristics and centers with different expertise.
Sometimes it is necessary for the reviewer to suggest that the author revise the manuscript to add more information about previous experience with the new technique, learning curve of the procedure, previous training, new devices and equipment necessary to the procedure, etc. This provides valuable information about limitations and strengths when the reader decides to reproduce the study in his or her own facilities. For instance, the positive efficacy of a procedure or a drug means that they work under ideal conditions, but this does not give us an answer as to whether the drug or treatment is effective or not in the real world. The questions to be answered by the reviewers are: 1) does the study evaluate efficacy or effectiveness of a technique or product; 2) is there enough information to reproduce the study elsewhere; 3 ) are the limitations and strengths of the study well defined; 4) are the results applicable, easy to implement, and can they probably modify the evolution of diseases; 5) can the reader generalize this study to his or her everyday work and their own patients; and 6) will the results improve patient care? 


\section{Reviewer's Checklists}

Surgeons commonly work with checklists. A checklist in the operating room ensures that everything needed for an operation has been considered in advance. Similarly, we also have checklists for clinical research to ensure that the people who conducted the clinical research really covered everything needed for a successful study. In Table 2, a checklist is presented to the reviewers to keep in mind the questions to be answered before sending the review to the editor.

\section{Conclusions}

Peer review is a remarkable process that relies on the trust and quality of the reviewers ensuring that published research is valid, significant, and original. The reviewer can detect bias, unsatisfactory design, and ethical problems in the study that may threaten the research, and he or she provides feedback to the authors to improve the manuscript. An appropriate review takes time because many things must be considered. The structure of the critical review presented allows one to weigh all the strengths and weaknesses of a submitted study, improving the quality of the review in less time.

\section{Acknowledgments}

We appreciate the support from the AO Foundation for Clinical Investigation and Documentation in preparation of this manuscript. This opinion piece is "patterned" after a prior publication of Dr. Falavigna's chapter "Formulating the question and PICOT," in Falavigna A, Jimènez Avila JM (eds): Education in Research: From the Idea to the Publication, ed 2. Caxias do Sul, Brazil: Educs, 2015, pp 45-52.

\section{References}

1. Bourne PE, Korngreen A: Ten simple rules for reviewers. PLOS Comput Biol 2:e110, 2006

2. Canadian Task Force on the Periodic Health Examination: The periodic health examination. Can Med Assoc J 121:1193-1254, 1979 (Erratum in Can Med Assoc J 122:163, 1980)
3. Chan AW: Out of sight but not out of mind: how to search for unpublished clinical trial evidence. BMJ 344:d8013, 2012

4. Cummings SR, Browner WS, Hulley SB: Conceiving the research question, in Hulley SB, Cummings SR, Browner WS, et al (eds): Designing Clinical Research: An Epidemiologic Approach, ed 2. Philadelphia: Lippincott Williams \& Wilkins, 2001, pp 17-23

5. Falavigna A: Formulating the question and PICOT, in Falavigna A, Jimènez Avila JM (eds): Education in Research: From the Idea to the Publication, ed 2. Caxias do Sul, Brazil: Educs, 2015, pp 45-52

6. Falavigna A, De Faoite D, Blauth M, Kates SL: Basic steps to writing a paper: practice makes perfect. Bangkok Med J 13:114-119, 2017

7. Glasziou P, Doll H: Was the study big enough? Two café rules. Evid Based Med 11:69-70, 2006

8. Hak DJ, Giannoudis P, Mauffrey C: Increasing challenges for an effective peer-review process. Eur J Orthop Surg Traumatol 26:117-118, 2016

9. Haynes RB: Clinical review articles. BMJ 304:330-331, 1992

10. Kehr P: Editorial. A new direction for EJOST! Eur J Orthop Surg Traumatol 24:1329, 2014

11. Rothman KJ: A potential bias in safety evaluation during open-label extensions of randomized clinical trials. Pharmacoepidemiol Drug Saf 13:295-298, 2004

12. Schulz KF, Altman DG, Moher D: CONSORT 2010 Statement: updated guidelines for reporting parallel group randomised trials. BMC Med 8:18, 2010

13. Scott-Lichter D, Editorial Policy Committee Council of Science Editors: CSE's White Paper on Promoting Integrity in Scientific Journal Publications, 2012 Update. 3rd Revised Edition. Wheat Ridge, CO: Council of Science Editors, 2012 (http://www.councilscienceeditors.org/wp-content/ uploads/entire_whitepaper.pdf) [Accessed June 23, 2017]

14. Shen C, Björk BC: 'Predatory' open access: a longitudinal study of article volumes and market characteristics. BMC Med 13:230, 2015

\section{Disclosures}

Dr. Kates reports receiving institutional research support from DePuy Synthes for resident research and travel support from the AO Foundation. 